Journal of Advanced Research in Fluid Mechanics and Thermal Sciences

Journal homepage: www.akademiabaru.com/arfmts.html ISSN: 2289-7879

\title{
Ultra-Thin Liquid Film with Shear and the Influences on Thermal Energy Transfer at Solid-Liquid Interfaces of Simple Liquid Methane in Contact With (110) Surface Structure of Face-Centred Cubic Lattice (FCC)
}

\author{
Abdul Rafeq Saleman ${ }^{1,},{ }^{*}$ Mohamad Shukri Zakaria ${ }^{1}$, Ridhwan Jumaidin ${ }^{2}$, Mohd Nazmin Maslan $^{3}$ \\ 1 Fakulti Kejuruteraan Mekanikal, Universiti Teknikal Malaysia Melaka, Malaysia, Hang Tuah Jaya, 76100 Durian Tunggal Melaka, Malaysia \\ 2 Fakulti Teknologi Kejuruteraan Mekanikal dan Pembuatan, Universiti Teknikal Malaysia Melaka, Hang Tuah Jaya, 76100 Durian Tunggal Melaka, \\ Malaysia \\ Fakulti Kejuruteraan Pembuatan, Universiti Teknikal Malaysia Melaka, Hang Tuah Jaya, 76100 Durian Tunggal Melaka, Malaysia
}

\section{ARTICLE INFO}

\section{Article history:}

Received 2 June 2021

Received in revised form 9 August 2021

Accepted 9 August 2021

Available online 28 September 2021

Keywords:

Thermal energy transfer; ultra-thin liquid film; solid-liquid interface

\section{ABSTRACT}

\begin{abstract}
Thermal energy transfer (TET) is the main performance of contact interfaces which has been studied at a molecular level. Several investigations on TET were accomplished, however, the influences of sheared liquid and liquid film thickness on TET have not been sufficiently examined. Thus, this paper analyses the influences of liquid film thickness on TET across solid-liquid (S-L) interfaces. Two liquid film thicknesses (Lz) of $30 \AA$ and $60 \AA$ h have been evaluated, and two shear directions ( $x$ - and $y$-directions) have been tested in the simulation system. It has been found that there is no significant difference in the density distribution of liquid regardless of the shear directions for the same Lz. However, there are differences in the density distribution of liquid between Lz of $30 \AA$ and $60 \AA$. Based on the results its suggests that, the cut-off of the temperature and velocity at the contact interfaces of solid and liquid is substantially influences by the shear applied to the liquid and the liquid film thickness of the simulation system. It is found that, there are a significant different in the thermal boundary resistance (TBR) for $L z$ of $30 \AA$ and $60 \AA$ for cases liquid sheared in the $x$ direction. Whereas TBR for $L z$ of $30 \AA$ and $60 \AA$ sheared in the $y$-direction have no significant difference. In conclusion, the TET is affected by the velocity cut-off at the contact interfaces of solid and liquid where larger velocity discontinuity exhibits higher TBR.
\end{abstract}

\section{Introduction}

The lubrication system is a system related to the contact interface between solid and liquid. The system has been utilised in vast engineering applications such as in lubrications and coatings [1-5], adsorption behaviour of liquid in contact with solid [6-7], In nano-fluid at the contact interfaces between nano-particle and base fluid [8-9], phase change material [10], Two phase flow gas and liquid [11] which is in relation to solid-liquid interfaces, surface treatment [12], water desalination

\footnotetext{
* Corresponding author.

E-mail address: rafeq@utem.edu.my
}

https://doi.org/10.37934/arfmts.87.3.2130 
process [13], thermal interface materials [14-16] and electronic cooling [17-18]. The contact interface between solid and liquid is represented as the interfaces of solid-liquid (S-L).

Based on past studies, the system of interfaces of S-L had failed without proper and well-designed thermal management system. It is crucial to identify the source of heat to rectify, optimise and control the performance of the overall system. Since these problems began at the molecular scale, it is important to understand the system at a molecular level. However, such problems are anomalous and cannot be predicted and tested based on conventional experiments and simulations. In recent years, such issues can now be solved with the use of available tools, such as the molecular dynamics simulation, which can reproduce the molecular scale phenomenon for more comprehensive analyses.

Several previous studies using the molecular dynamics simulation associated to thermal energy transfer (TET) across the interfaces of $S-L$ have been conducted [17-19-24]. They examined the molecular interaction forces between solid and liquid [25], shearing applied to the liquid [26], influences of molecular length on TET [19-27] as well as the hydrophilic and hydrophobic behaviours of liquid [28-29]. However, research associated with TET with the influence of shear at ultra-thin liquid film have not been conducted. In the previous study the Xiao Liu et al., [30] (2020) have look into the influence of liquid film thickness but not at both the influences liquid film thickness and effect of shear liquid film. Thus, this paper investigates the influence of different liquid film thicknesses towards TET at the contact interfaces of S-L by the method known as non-equilibrium molecular dynamics (NEMD) simulation. In addition, two different liquid film thicknesses as well as two shear directions have been applied to the simulation system. And since, the surface structure of the solid has different surface roughness at different shear direction [27].

\section{Methodology}

\subsection{Model}

As shown in Figure 1 model is consisted enclosed two parallel solid walls, where liquid film is trapped in it. Gold ( $\mathrm{Au}$ ) was used for the solid walls and liquid methane was used for the liquid. The solid walls have the surface structure of (110) surfaces from the face centred cubic (FCC) lattice, contacting liquid methane. Both sides of the solid walls, consisted of 10 layers of (110) surface. Whereas the liquid region, liquid methane was randomly distributed. Two liquid film thicknesses $\left(L_{z}\right)$ of $30 \AA$ and $60 \AA$ were used for the simulation model. The contact of the solid and liquid on both sides of the model is referred as the interfaces of the S-L. The model has a size of approximately $40 \AA$ in both $x$ - and $y$-axis, no matter what of $L_{z}$. For the $z$-axis, the size was approximately $120 \AA$ for $L_{z}$ of $60 \AA$ and $90 \AA$ for $L_{z}$ of $30 \AA$. To ensure that the simulation model does not fluctuate during simulation, the $z$-direction was fixed in the simulation system. Whereas in $x$ - and $y$-directions a periodic boundary condition (PBC) was applied. 


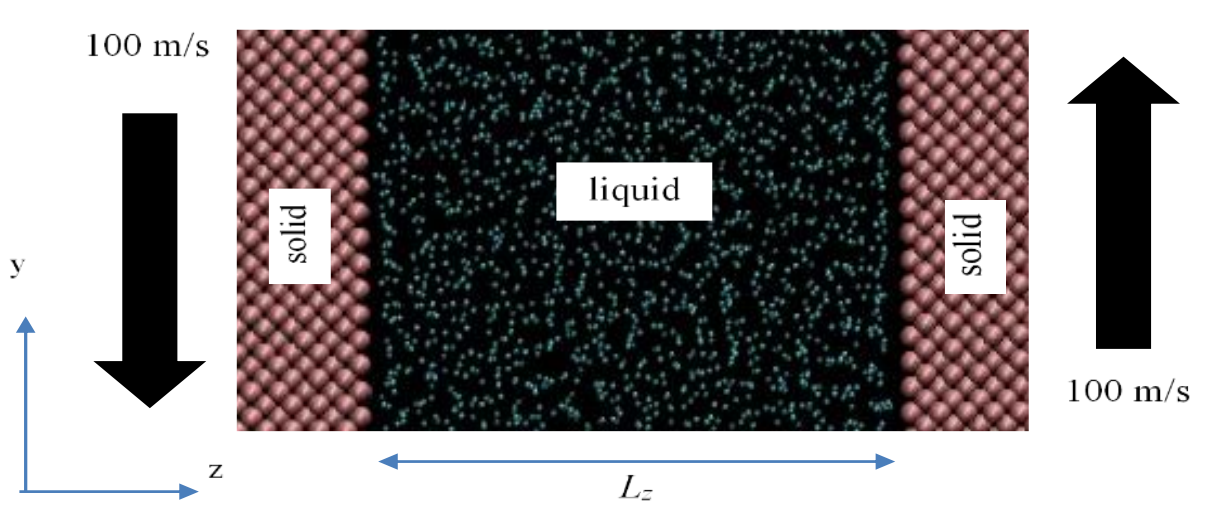

Fig. 1. Twov parallel solid walls in contact with liquid film in between them

\subsection{Potential Function}

The interaction force between the solid wall molecules of Au was modelled using the Morse potential [3132]. United atom (UA) model has been utilized to model the liquid methane. In the UA model, the four atoms of Hydrogen and a single atom of Carbon is group as one single pseudo-atom. The interaction forces of each pseudo-atom of UA were displayed using the Lennard Jones (12-6) potential [24-27-33]. Next, Lorentz-Bertholet combining rules were utilized to model the interaction forces between pseudo-atom of liquid and atom of solid. A cut-off radius of $12.0 \AA$ were applied to all interaction forces in the simulation. The same molecule model and potential functions have been utilised in previous studies [24-27-30-33].

\subsection{Simulation Details}

The reversible references propagator algorithm ( $r$-RESPA) was employed as the time integration method [34]. Initially, the temperature of the simulation system was raised to the $0.7 T_{c}$ of the targeted temperature for 1 to 2 million time steps. Then, the temperature of $0.7 T_{c}$ was controlled to acquire a uniform temperature throughout the simulation system for 2 to 3 million time steps. Next, the shear setup was applied to the simulation system where a constant and opposite speed of 100 $\mathrm{m} / \mathrm{s}$ was imposed to the two parallel solid walls. Figure 1 shows the simulation setup. The same speed was applied in a previous study Saleman et al., [19] (2017). Since the surface structure of the FCC lattice (110) is unsymmetrical (Figure 2), two shear directions were applied to the simulation system in the $x$ - and $y$-directions. The shear setup was conducted and run for another 5 million time steps. Finally, the data acquisition was run for 10 million time steps.

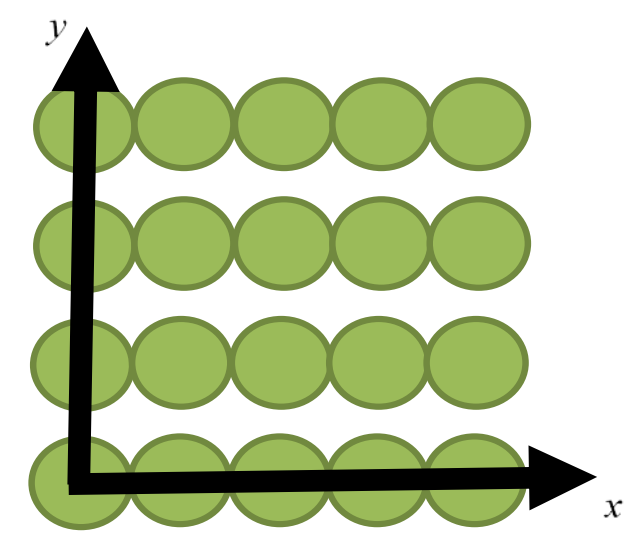

Fig. 2. Surface structure of FCC 110 surfaces 


\section{Results}

\subsection{Density Profiles}

Density profiles play an important role in determining the liquid characteristics of liquid adsorption behaviour on a solid and the orientation of liquid molecules. In this study, the density distribution was calculated by splitting the simulation system into 6000 number of blocks in the $z$-direction. The density distribution for each one of the blocks was calculated according to the number of molecules present in each block. Saleman et al., [19] (2017) had also employed the same method for determining the definition and calculation of the blocks to acquire the density profile. Figure 3 presents the density profile for $L_{z}$ of $60 \AA$ and $30 \AA$ sheared in the $x$ - and $y$ directions.

As to make sure that the density profile for all cases is comparable, the peak height of the nearest adsorption layer to the interfaces of $S-L$ was controlled in such that the peak heights for all cases are approximately the same. Since the density profiles for the liquid sheared in the $x$ - and $y$-directions exhibited similar profiles, only the density profiles of $L_{z}$ of $60 \AA$ and $30 \AA$ sheared in the $x$-direction are displayed in Figure 3.

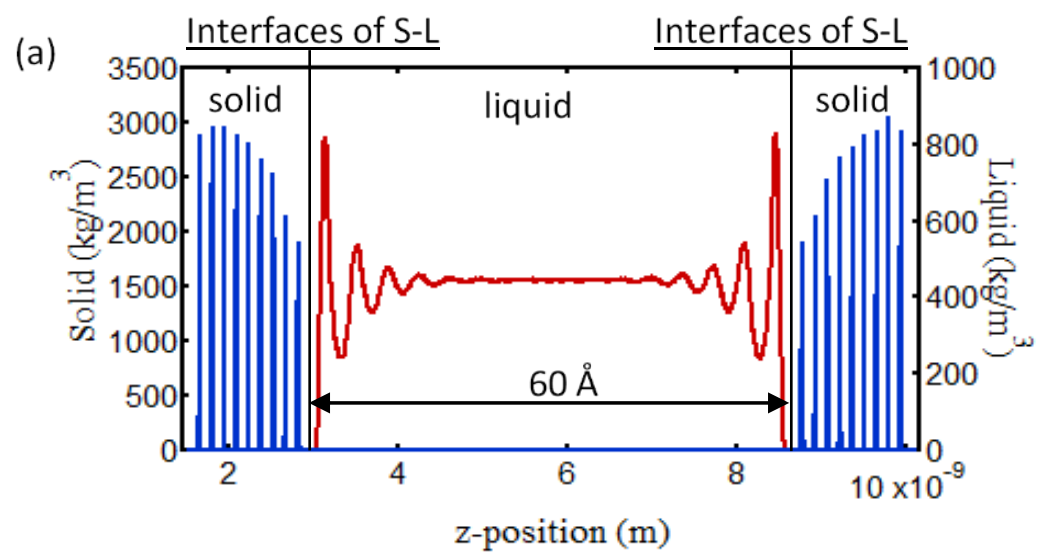

(b)

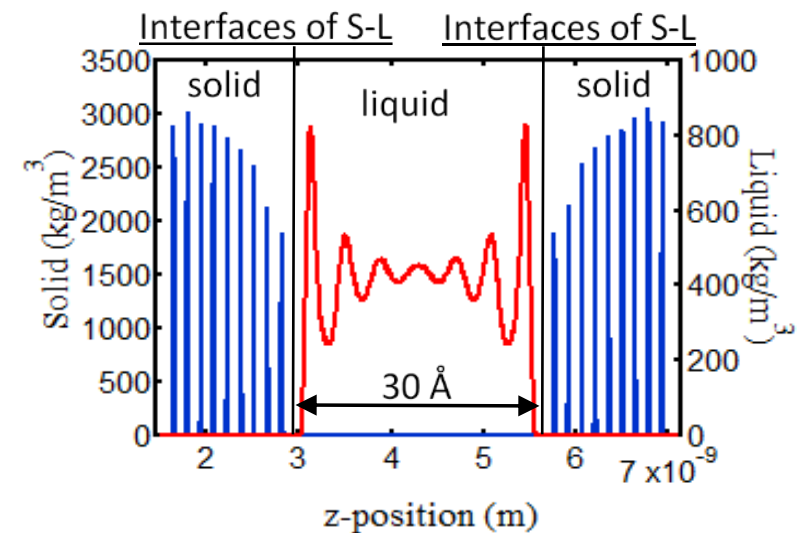

Fig. 3. Density distributions for (a) $60 \mathrm{~A}$ and (b) $30 \mathrm{~A}$ sheared in $\mathrm{X}$ and $y$-directions

. Based on the figure, the density profiles for the system with liquid $L_{z}$ of $60 \AA$ oscillated near the interfaces of $S-L$ for both shear directions, and a flat line was observed at the centre of the system. The same density profile was also noticed in several previous studies [19-33]. The same oscillation near the interfaces of $S-L$ was also recorded for the system with liquid $L_{z}$ of $30 \AA$ in both shear directions, but no flat line was observed at the centre of the density distribution. The non-existence 
of the flat line at the centre of the density distribution for $L_{z}$ of $30 \AA$ indicates that there exists an interaction force in the liquid by the solid walls. As such, the interaction force may influence the TET across the simulation system.

\subsection{Temperature Profiles}

Figure 4 shows that the simulation system was divided based on the density profiles for calculating the temperature profiles. The temperature profiles were calculated from the velocity of each molecule in each block. Both microscopic velocity and macroscopic velocity were present in this simulation. To calculate the temperature profiles, only the microscopic velocity in the $x-, y$ - and $z$ directions were considered.

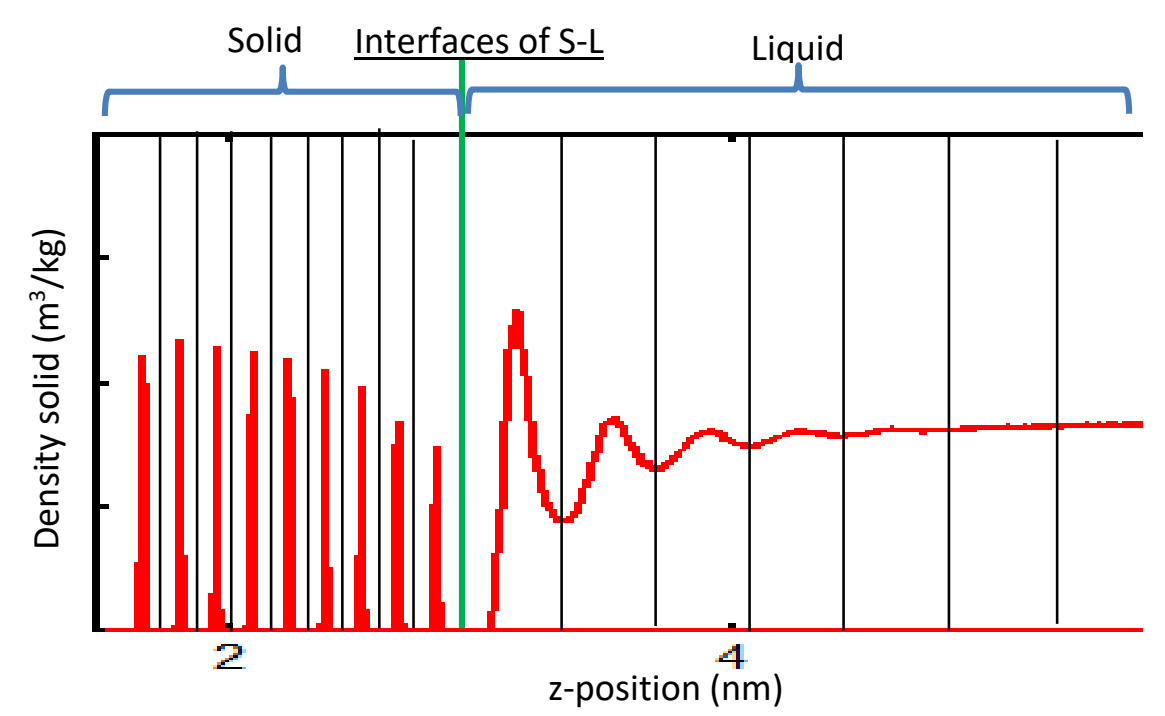

Fig. 4. Definition of slab for temperature distributions

Figure 5 presents the temperature distributions for the simulation system with liquid film thicknesses of $60 \AA$ and $30 \AA$ sheared in both directions. Temperature discontinuity (TD) was observed at the interfaces of $S-L$ for all cases of the simulation system. The TD is calculated by extrapolating the temperature of solid and liquid in the $z$-direction to the point where the interfaces of $S-L$ is located. In this study the location of the interfaces of S-L is assume exactly at the centre between solid and liquid layers. The temperature difference between the solid and liquid is then calculated. Table 1 displays the TD for $L_{z}$ of $30 \AA$ and $60 \AA$ sheared in both directions. The TD was higher for cases sheared in the $y$-direction regardless of the liquid film thickness. Based on Table 1 , the TD for $30 \AA$ sheared in the $y$-direction was the highest, followed by TD for $60 \AA$ sheared in the $y$ direction and $30 \AA$ and $60 \AA$ sheared in the $x$-direction. The results of the temperature profiles suggest that the liquid film thickness does have a significant influence on the TD at the interfaces of S-L.

Table 1

Heat flux (HF) and Thermal boundary resistance (TBR) for $30 \AA$ and $60 \AA$ shear in $\mathrm{x}$ and $\mathrm{y}$-directions

\begin{tabular}{lllll}
\hline Shear direction & $x$-direction & \multicolumn{3}{c}{$y$-direction } \\
\hline $\mathrm{L}_{2}$ & 30 & 60 & 30 & 60 \\
$\mathrm{TJ}(\mathrm{K})$ & 14.35 & 10.65 & 34.91 & 19.34 \\
$\mathrm{VJ}(\mathrm{K})$ & 62.4 & 47.3 & 11.9 & 7.7 \\
$\mathrm{HF}(\mathrm{WM})$ & 102.8 & 94.6 & 334.8 & 183.8 \\
$\mathrm{TBR} \times 10^{7}$ & 1.395 & 1.132 & 1.043 & 1.052 \\
\hline
\end{tabular}


(a)

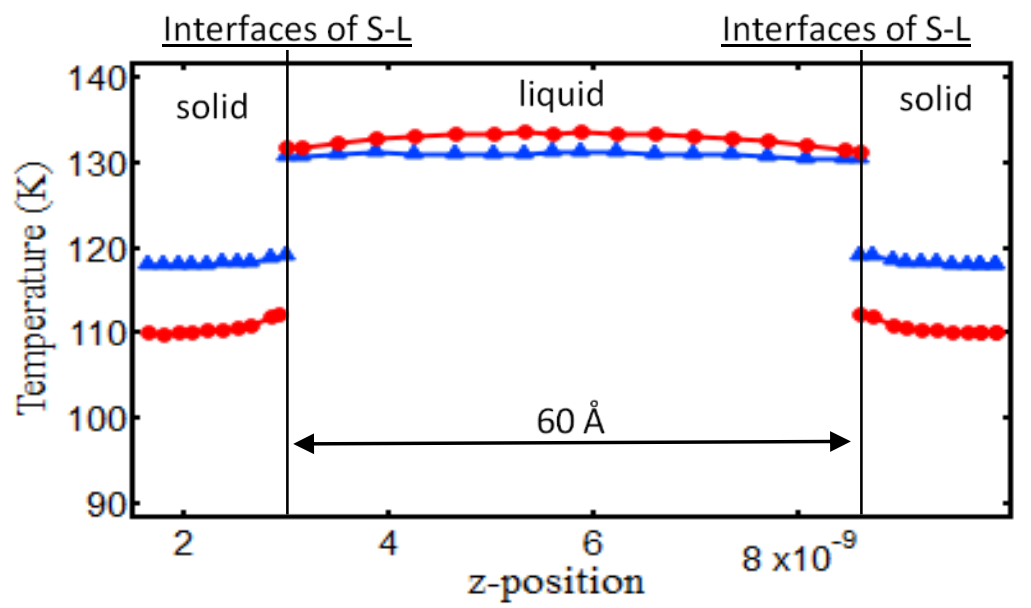

(b)

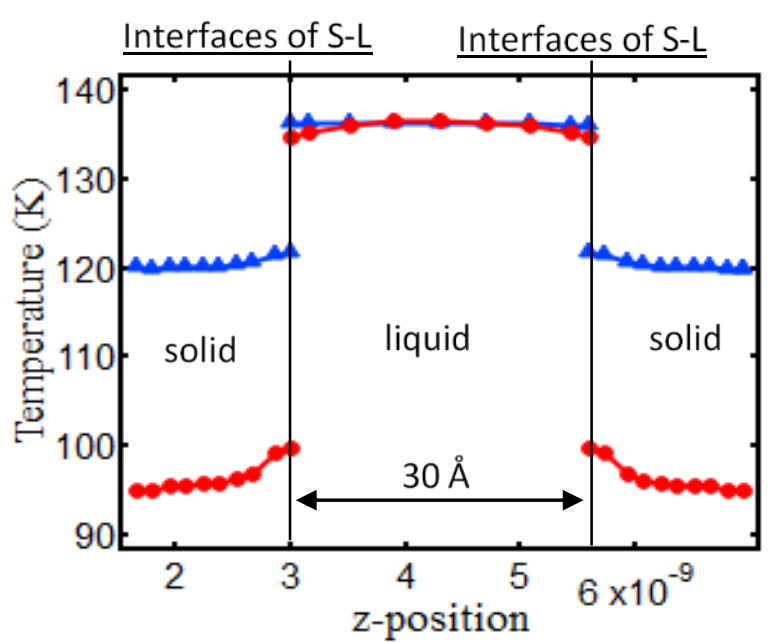

Fig. 5. Temperature distributions for (a) $60 \AA$ and (b) $30 \AA$ sheared in $x$ - (blue) and $y$ directions (red)

\subsection{Velocity Profiles}

The same definition of blocks in Figure 4 was also utilised to calculate the velocity profiles for the system with liquid $L_{z}$ of $30 \AA$ and $60 \AA$ sheared in both directions. In the calculation of velocity profiles, only macroscopic velocity was considered. As shown in Figure 6, velocity discontinuity (VD) was observed at the interfaces of $S-L$ for all cases of the simulation system. The behaviour of VD was also detected in a previous study by Saleman et al., [19] (2017). In this section, the same method for calculating TD was utilised to calculate VD. Table 1 presents the value of VD for the simulation model with liquid $L_{z}$ of $30 \AA$ and $60 \AA$ for both shear directions. Based on the results, the VD with liquid film thickness of $30 \AA$ sheared in the $x$-direction had the highest value in the system, followed by the liquid film thickness of $60 \AA$ sheared in the $x$-direction and then the system with liquid film thickness of 30 $\AA$ sheared in the $y$-direction. The lowest VD was for $L_{z}$ of $60 \AA$ sheared in the $y$-direction. It has been generally observed that the VD for the system with liquid $L_{z}$ of $30 \AA$ and $60 \AA$ sheared in the $y$-direction have a small difference between them, approximately $4.2 \mathrm{~K}$. While the VD of the system with liquid film thicknesses of $30 \AA$ and $60 \AA$ sheared in the $x$-direction have a larger value in comparison, with approximately $15.1 \mathrm{~K}$ difference between them. This behaviour is due to the surface structure of the FCC (110) surface, as observed in previous studies [19-24-27]. Past research had mentioned that 
there exists peaks and valleys on the surface of (110). Some liquid molecules can get trapped in the valley of the solid surface and as a result, the VD decreases. However, the shear direction can influence the VD in the parallel direction to the peak and valley of the FCC (110) surface. If the shear applied to the system is in the parallel direction, the liquid molecules can easily get pulled, creating a larger VD compared to shear applied in the perpendicular direction to the peak and valley of the surface. Based on the observation of the temperature profiles and velocity profile, it has been suggested that the thin liquid influences the VD characteristics at the interfaces of $S-L$ where the smaller liquid film thickness has higher values of VD.

(a)

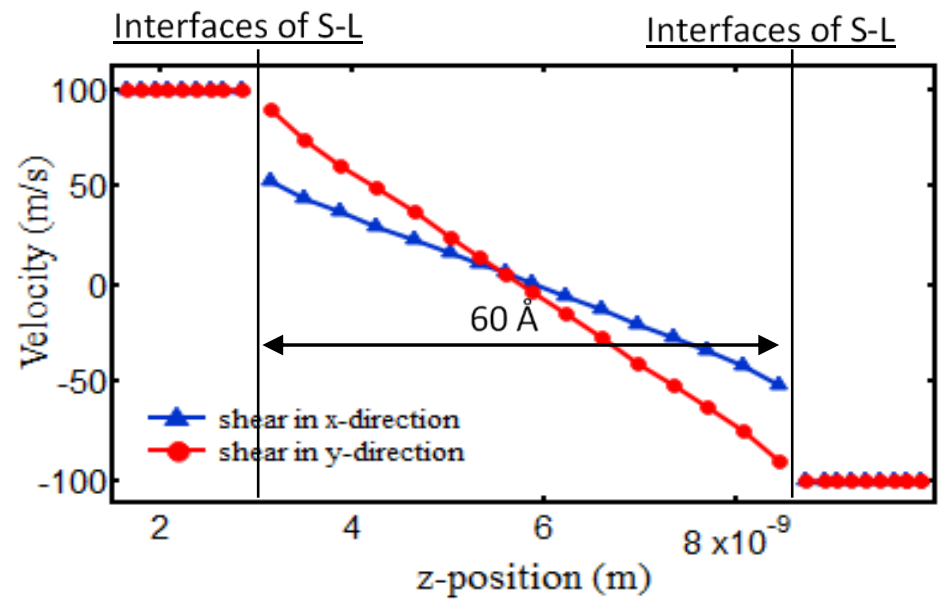

(b)

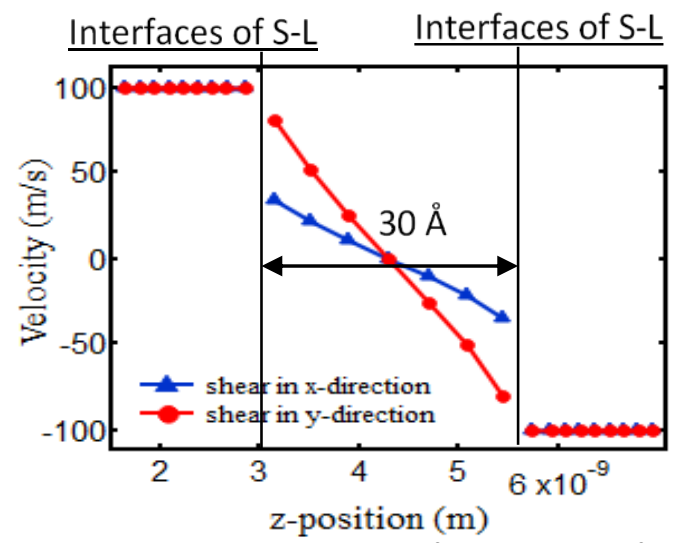

Fig. 6. Velocity distributions for (a) $60 \AA$ and (b) $30 \AA$ sheared in $x$ - (blue) and y-directions (red)

Table 1 displays the heat flux (HF) value all over the simulation system and thermal boundary resistance (TBR) at the interfaces of $S-L$ for the simulation system of $30 \AA$ and $60 \AA$ sheared in both directions. The HF was different for each case of the simulation system. This is expected since the VD is different for each case of the simulation system. However, the order of HF varied from VD where the increasing order of heat flux is: $60 \AA$ sheared in the $x$-direction, followed by $30 \AA$ sheared in the $x$-direction, $60 \AA$ sheared in the $y$-direction and the highest, $30 \AA$ sheared in the $y$-direction. To justify the factor that influences VD, TD and HF, the TBR was calculated. TBR is the ratio of TD and HF at the interfaces of S-L.

Figure 7 presents the TBR versus $L_{z}$ for the simulation system sheared in both directions. The TBR for the cases of the simulation system sheared in the $x$-direction decreased as the $L_{z}$ increased. This is due to the high TD, elevated VD and low HF value near the interfaces of S-L. Although the TD, VD, 
and HF values are different between the two cases, the TBR for the cases of $30 \AA$ and $60 \AA$ sheared in the $y$-direction presented an approximately similar value, as shown in Figure 7 . This is due to the factor of adsorption of the liquid molecules on the solid surfaces, which was also observed in previous studies by A. R. b. Saleman et al., [19] (2017), A. R. bin Saleman et al., [27] (2017) and Saleman et al., [33] (2018). The liquid molecules are adsorbed in the valley of the FCC (110) surface which help in the TET. In short, the result suggests that the shear liquid and liquid film thickness does not influence the TBR regardless for the cases liquid sheared in y-direction. However as reported by Xiao Liu et al., [30] (2020) the TBR increase with the increase in liquid film thickness. Such differences suggests that the shear liquid influences the characteristics of TBR at the contact interfaces in relation to VD.

According to the results however, if the effect of the surface structure is neglected, this would also suggest that the TBR is significantly influenced by VD at the interfaces of S-L where large VD creates higher TBR.

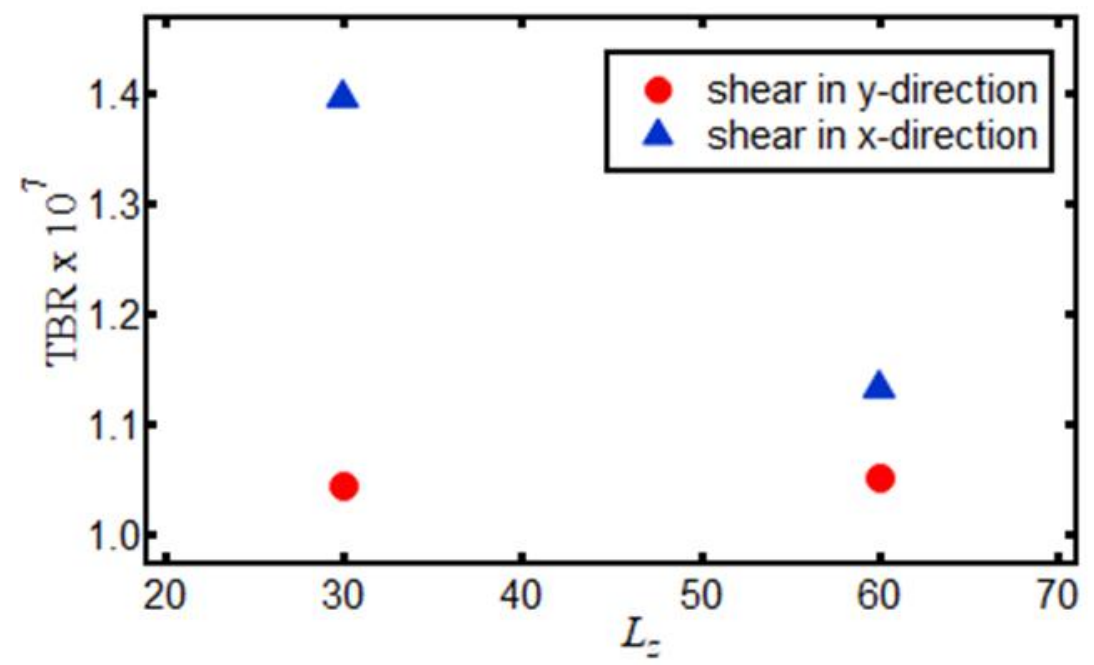

Fig. 7. Thermal boundary resistance versus $L z$ for simulation system shear in $\mathrm{x}$ - and $\mathrm{y}$-direction

\section{Conclusions}

In this study, the influences of liquid film thickness $\left(L_{z}\right)$ and shear on the qualities of thermal energy transfer (TET) at the interfaces of $S-L$ were investigated. The influence of liquid film thickness was investigated based on the liquid $L_{z}$ of $30 \AA$ and $60 \AA$ where both simulations modelled were sheared in both the $x$ - and $y$-directions. The results suggests that the decrease in liquid $L_{z}$ increases the temperature discontinuity (TD) and velocity discontinuity (VD) at the interfaces of $S-L$ regardless of the shear direction. Although the liquid film thickness significantly influences the TD and VD, the thermal boundary resistance (TBR) for the case of liquid sheared in the $y$-direction is approximately equal for $L_{z}$ of both $30 \AA$ and $60 \AA$. Based on previous study [30] and present simulation results, suggests that the TBR is influence by the sheared liquid. As for the simulation system sheared in the $x$-direction, it was found that there is a large increment in TBR for $L_{z}$ of $30 \AA$ compared to $60 \AA$. This behaviour was due to the surface structure of the FCC (110) surface and the shear directions. The results concluded that the liquid film thickness significantly influences the TD and VD. However, for TBR, the liquid film thickness influences the system sheared in the $x$-direction but not in the $y$-direction. Such behaviour is due to the arrangement of the surface structure of (110) which can either be in the parallel direction or in the perpendicular direction with the direction of shear. 


\section{Acknowledgement}

The authors would like to thank Assoc. Prof. Gota Kikugawa from the Institute of Fluid Science, Tohoku University since he developed the software used to run the simulations.

\section{References}

[1] Septiadi, Wayan Nata, Ketut Astawa, I. Gusti Ayu Pristha Arvikadewi, David Febraldo, and Gerardo Janitra Puriadi Putra. "Boiling Phenomenon of Graphene Nano-Coating Wick Heat Pipe." Evergreen 7, no. 2 (2020): $297-302$. https://doi.org/10.5109/4055236

[2] Rizky, Ruliandini, and Takashi Tokumasu. "Assessing hBN nanoparticles stability in trimethylolpropane triester based biolubricants using molecular dynamic simulation." Evergreen 7, no. 2 (2020): $234-239$. https://doi.org/10.5109/4055225

[3] Ligner, G., M. Sidqi, J. Jagiello, H. Balard, and E. Papirer. "Characterization of specific interactions capacity of solid surfaces by adsorption of alkanes and alkenes. Part II: Adsorption on crystalline silica layer surfaces." Chromatographia 29, no. 1-2 (1990): 35-38. https://doi.org/10.1007/BF02261136

[4] Gao, Gengyuan, Zhongwei Yin, Dan Jiang, and Xiuli Zhang. "Numerical analysis of plain journal bearing under hydrodynamic lubrication by water." Tribology International $75 \quad$ (2014): https://doi.org/10.1016/j.triboint.2014.03.009

[5] Apóstolo, Rui FG, Georgia Tsagkaropoulou, and Philip J. Camp. "Molecular adsorption, self-assembly, and friction in lubricants." Journal of Molecular Liquids 277 (2019): 606-612. https://doi.org/10.1016/j.molliq.2018.12.099

[6] Ghani, Nik Rashida Nik Abdul, and Mohammed Saedi Jami. "Dynamic Adsorption of Lead by Novel Graphene Oxidepolyethersulfone Nanocomposite Membrane in Fixed-bed Column." Journal of Advanced Research in Experimental Fluid Mechanics and Heat Transfer 2, no. 1 (2020): 1-9.

[7] Ridassepri, Arikasuci Fitonna, Fitria Rahmawati, Kinkind Raras Heliani, Jin Miyawaki, and Agung Tri Wijayanta. "Activated carbon from bagasse and its application for water vapor adsorption." Evergr. Jt. J. Nov. Carbon Resour. Green Asia Strateg 7 (2020): 409-416. https://doi.org/10.5109/4068621

[8] Jahan, Sultana, M. Ferdows, M. D. Shamshuddin, and Khairy Zaimi. "Effects of Solar Radiation and Viscous Dissipation on Mixed Convective Non-Isothermal Hybrid Nanofluid over Moving Thin Needle." Journal of Advanced Research in Micro and Nano Engineering 3, no. 1 (2021): 1-11.

[9] Mohd Yatim, Muyassarah Syahirah, Irnie Azlin Zakaria, Mohamad Fareez Roslan, and Wan Ahmad Najmi Wan Mohamed. "Heat transfer and pressure drop characteristics of hybrid $\mathrm{Al}_{2} \mathrm{O}_{3}-\mathrm{SiO}_{2}$." Journal of Mechanical Engineering (JMechE) 8, no. 2 (2021): 145-159.

[10] WafirulHadi, Mohamad, Titin Trisnadewi, and Nandy Putra. "Thermal Management System Based on Phase Change Material (PCM) and Heat Pipe in Lithium-ion Electric Vehicle Batteries." Journal of Advanced Research in Experimental Fluid Mechanics and Heat Transfer 3, no. 1 (2021): 26-35.

[11] Sukamta, Sukamta. "Computational fluid dynamics (CFD) and experimental study of two-phase flow patterns gasliquid with low viscosity in a horizontal capillary pipe." CFD Letters 11, no. 8 (2019): 16-23.

[12] Fadzullah, SH Sheikh Md, M. M. Nasaruddin, Z. Mustafa, W. A. W. A. Rahman, G. Omar, M. A. Salim, and M. R. Mansor. "The effect of chemical surface treatment on mechanical performance of electrically conductive adhesives." J. Novel Carbon Res. Sci. Green Asia Strategy 7 (2020): 444-451. https://doi.org/10.5109/4068625

[13] Cohen-Tanugi, David, and Jeffrey C. Grossman. "Water desalination across nanoporous graphene." Nano letters 12 , no. 7 (2012): 3602-3608. https://doi.org/10.1021/nl3012853

[14] Kim, Bo Hung, Ali Beskok, and Tahir Cagin. "Thermal interactions in nanoscale fluid flow: molecular dynamics simulations with solid-liquid interfaces." Microfluidics and Nanofluidics 5, no. 4 (2008): 551-559. https://doi.org/10.1007/s10404-008-0267-7

[15] Liang, Zhi, and Hai-Lung Tsai. "Thermal conductivity of interfacial layers in nanofluids." Physical Review E 83, no. 4 (2011): 041602. https://doi.org/10.1103/PhysRevE.83.041602

[16] Li, Xiaobo, and Ronggui Yang. "Effect of lattice mismatch on phonon transmission and interface thermal conductance across dissimilar material interfaces." Physical Review B 86, no. 5 (2012): 054305. https://doi.org/10.1103/PhysRevB.86.054305

[17] Tang, Yunqing, Junchao Li, Xiaoju Wu, Qiaoya Liu, Yu Liu, and Ping Yang. "Tunable thermal property in edge hydrogenated AA-stacked bilayer graphene nanoribbons." Applied Surface Science 362 (2016): 86-92. https://doi.org/10.1016/i.apsusc.2015.11.179

[18] Hooch Antink, Wytse, Yejung Choi, Kwang-dong Seong, Jong Min Kim, and Yuanzhe Piao. "Recent Progress in Porous Graphene and Reduced Graphene Oxide-Based Nanomaterials for Electrochemical Energy Storage Devices." Advanced Materials Interfaces 5, no. 5 (2018): 1701212. https://doi.org/10.1002/admi.201701212

[19] bin Saleman, Abdul Rafeq, Hari Krishna Chilukoti, Gota Kikugawa, Masahiko Shibahara, and Taku Ohara. "A 
molecular dynamics study on the thermal energy transfer and momentum transfer at the solid-liquid interfaces between gold and sheared liquid alkanes." International Journal of Thermal Sciences 120 (2017): 273-288. https://doi.org/10.1016/j.ijthermalsci.2017.06.014

[20] Merabia, Samy, Julien Lombard, and Ali Alkurdi. "Importance of viscoelastic and interface bonding effects in the thermal boundary conductance of solid-water interfaces." International Journal of Heat and Mass Transfer 100 (2016): 287-294. https://doi.org/10.1016/j.ijheatmasstransfer.2016.04.043

[21] Vo, Truong Quoc, and BoHung Kim. "Interface thermal resistance between liquid water and various metallic surfaces." International Journal of Precision Engineering and Manufacturing 16, no. 7 (2015): 1341-1346. https://doi.org/10.1007/s12541-015-0176-0

[22] Nakano, Takeo, Gota Kikugawa, and Taku Ohara. "Molecular heat transfer in lipid bilayers with symmetric and asymmetric tail chains." Journal of heat transfer 135, no. 6 (2013). https://doi.org/10.1115/1.4023572

[23] Vo, Truong Quoc, Murat Barisik, and BoHung Kim. "Atomic density effects on temperature characteristics and thermal transport at grain boundaries through a proper bin size selection." The Journal of chemical physics 144, no. 19 (2016): 194707. https://doi.org/10.1063/1.4949763

[24] Saleman, Abdul Rafeq, Fudhail Abdul Munir, Mohd Rody Mohammad Zin, Mohd Shukri Yob, Gota Kikugawa, and Taku Ohara. "Heat Transport at Solid-Liquid Interfaces between FaceCentered Cubic Lattice and Liquid Alkanes." Journal of Advanced Research in Fluid Mechanics and Thermal Sciences 44, no. 1 (2018): 123-130.

[25] Torii, Daichi, Taku Ohara, and Kenji Ishida. "Molecular-scale mechanism of thermal resistance at the solid-liquid interfaces: influence of interaction parameters between solid and liquid molecules." Journal of Heat Transfer 132, no. 1 (2010). https://doi.org/10.1115/1.3211856

[26] Martini, A., A. Roxin, R. Q. Snurr, Q. Wang, and S. Lichter. "Molecular mechanisms of liquid slip." Journal of Fluid Mechanics 600 (2008): 257-269. https://doi.org/10.1017/S0022112008000475

[27] Bin Saleman, Abdul Rafeq, Hari Krishna Chilukoti, Gota Kikugawa, Masahiko Shibahara, and Taku Ohara. "A molecular dynamics study on the thermal transport properties and the structure of the solid-liquid interfaces between face centered cubic (FCC) crystal planes of gold in contact with linear alkane liquids." International Journal of Heat and Mass Transfer 105 (2017): 168-179. https://doi.org/10.1016/j.ijheatmasstransfer.2016.09.069

[28] Ge, Zhenbin, David G. Cahill, and Paul V. Braun. "Thermal conductance of hydrophilic and hydrophobic interfaces." Physical review letters 96, no. 18 (2006): 186101. https://doi.org/10.1103/PhysRevLett.96.186101

[29] Wang, Yun, and Mehernosh Gundevia. "Measurement of thermal conductivity and heat pipe effect in hydrophilic and hydrophobic carbon papers." International Journal of Heat and Mass Transfer 60 (2013): $134-142$. https://doi.org/10.1016/j.ijheatmasstransfer.2012.12.016

[30] Liu, Xiao, Donatas Surblys, Yoshiaki Kawagoe, Abdul Rafeq Bin Saleman, Hiroki Matsubara, Gota Kikugawa, and Taku Ohara. "A molecular dynamics study of thermal boundary resistance over solid interfaces with an extremely thin liquid film." International Journal of Heat and Mass Transfer 147 (2020): 118949. https://doi.org/10.1016/j.ijheatmasstransfer.2019.118949

[31] T. Morse and S. H. Oscillator. "The Morse Potential." Energy 2 (2003): 2-4.

[32] Kikugawa, Gota, Taku Ohara, Tohru Kawaguchi, Ikuya Kinefuchi, and Yoichiro Matsumoto. "A molecular dynamics study on heat conduction characteristics inside the alkanethiolate SAM and alkane liquid." International Journal of Heat and Mass Transfer 78 (2014): 630-635. https://doi.org/10.1016/j.ijheatmasstransfer.2014.07.040

[33] Saleman, Abdul Rafeq Bin, Fudhail Abdul Munir, Mohd Fadzli Bin Abdollah, Gota Kikugawa, and Taku Ohara. "Comparison of the characteristic of heat transport between non-shear and shear systems at solid-liquid (SL) interfaces." Proceedings of Mechanical Engineering Research Day 2018 (2018): 270-272.

[34] Tuckerman, M. B. B. J. M., Bruce J. Berne, and Glenn J. Martyna. "Reversible multiple time scale molecular dynamics." The Journal of chemical physics 97, no. 3 (1992): 1990-2001. https://doi.org/10.1063/1.463137 